\title{
Ensayando una política pública de asistencia mental: El movimiento de higiene mental en Chile, 1891-1948
}

Essaying a public policy of mental assistance: The mental hygiene movement in Chile, 1891-1948

Claudia ARAYA IBACACHE 1

Universidad Católica de Chile, Chile c.arayaibacache@gmail.com

\section{Resumen}

Desde la inauguración de la Casa de Orates en 1852 y durante toda la segunda mitad de ese siglo, los alienistas abogaron ante el Gobierno por la medicalización de la Institución. A este grupo le siguió, a partir de fines del siglo XIX, otro grupo de psiquiatras que apelaron nuevamente a la autoridad gubernamental buscando esta vez la transformación del asilo en hospital psiquiátrico. La investigación que presento cubre el período que va entre las propuestas de este segundo grupo y el año 1948, cuando se organiza la Asociación Chilena Pro-Salud Mental, filial de su homónima latinoamericana. Se presenta un amplio arco de iniciativas que va desde la promoción de instancias asociativas hasta la formulación de proyectos de institucionalización de los nuevos modelos asistenciales. Postulo como hipótesis que, a partir del modelo asistencial preventivo, propuesto para el desarrollo de la higiene mental, surgieron los primeros discursos en torno a la necesidad de una política pública de salud mental en Chile.

Doctora en Historia, Investigadora Programa de Estudios Médicos Humanísticos de la Universidad Católica de Chile (PEMHUC), Facultad de Medicina, Universidad Católica de Chile.

Esta investigación forma parte del Proyecto Fondecyt Posdoctorado №3180429, “Antecedentes, propuestas y formulación de políticas de salud mental en Chile, 1940-1990”.

Claudia ARAYA IBACACHE

Ensayando una política pública de asistencia mental: El movimiento de higiene mental en Chile, 18911948

Sur y Tiempo. Revista de Historia de América, №3, enero-junio 2021, pp. 86-98.

ISSN 2452-574X

DOI: $10.22370 /$ syt.2021.3.2700 
Palabras clave: Salud pública; Salud mental; Higiene mental; Chile; Hospital Psiquiátrico.

\begin{abstract}
Since the inauguration of the House of Orates in 1852 and throughout the second half of that century, the alienists advocated before the Government for the medicalization of the Institution. This group was followed, starting in the late nineteenth century, by another group of psychiatrists who appealed again to government authority, this time seeking to transform the asylum into a psychiatric hospital. The research that I present covers the period between the proposals of this second group and the year 1948, when the Chilean Association Pro-Mental Health was organized, a subsidiary of its Latin American namesake. A wide range of initiatives is presented, ranging from the promotion of associative instances to the formulation of projects to institutionalize the new healthcare models. I suggest as a hypothesis that, from the preventive care model, proposed for the development of mental hygiene, the first discourses arose around the need for a public mental health policy in Chile.
\end{abstract}

Keywords: Public health; Mental health; Mental higiene; Chile; Psychiatric Hospital.

\title{
Introducción
}

Esta propuesta busca analizar el papel que jugó el desarrollo de la higiene mental en los primeros intentos de transformación del modelo asilar de asistencia. Postulamos como hipótesis que, a partir de los cambios propuestos al modelo, surgieron los primeros lineamientos de lo que podríamos considerar una política pública en torno a la asistencia mental en Chile. Hasta avanzado el siglo XX, la asistencia mental en Chile tuvo un marcado carácter asilar (Marconi, 1998; Araya, 2018), a lo que se suma un pobre desarrollo de la legislación sanitaria en el mismo campo y una evidente preocupación por el tema en el discurso político y social del país. La principal institución de asistencia mental en Chile se fundó en 1852, en Santiago, con el nombre de Casa de Orates de Nuestra Señora de Los Ángeles.

La historiografía sanitaria tradicional ha construido la idea de que desde fines del siglo XIX la evolución de las instituciones sanitarias ha sido marcada por la continuidad histórica, como expresión de madurez de la conciencia cívica (Molina, 2010). Sin embargo, en el caso que nos ocupa, la evidencia muestra que estamos ante una diversidad de líneas de desarrollo que transcurren a través de derroteros también distintos y tributarios de procesos complejos y muchas veces disímiles entre sí. A diferencia del resto de las instituciones sanitarias del país, dependientes de la Junta Nacional de Beneficencia, la Casa de Orates dependía directamente del Ministerio del 
Interior, encargado de la seguridad y el orden público. Desde su inauguración y durante todo lo que quedaba del siglo, los alienistas abogaron ante el Gobierno chileno por la medicalización de la Institución con resultados deficientes (Camus, 1993).

A este grupo de médicos le siguió, a partir de fines del siglo XIX, otro grupo de psiquiatras que apelaron nuevamente a la autoridad gubernamental buscando esta vez la transformación del asilo en hospital psiquiátrico, entre otras demandas. Esta investigación cubre el período que va entre las propuestas de este segundo grupo y el año 1948, cuando se organiza la Asociación Chilena Pro-Salud Mental, filial de su homónima latinoamericana. Durante este período, psiquiatras, neurólogos y otros profesionales que no pertenecían al ámbito sanitario, emprendieron una serie de iniciativas, tanto individuales como colectivas, tendientes a organizar la higiene mental en el país, un arco que va desde la promoción de instancias asociativas hasta la formulación de proyectos de institucionalización de los nuevos modelos asistenciales. Una de estas últimas fue la creación de la Asociación Chilena de Higiene Mental2 en 1931, un año después de la realización del Primer Congreso Internacional de Higiene Mental, en Washington.

En el primer apartado presentamos las propuestas de los médicos Manuel Segundo Beca, Germán Greve y Jerónimo Letelier, las que cubren un período que va desde la última década de 1890 hasta la década de 1930. Las hemos seleccionado por poco conocidas, a pesar de que son producto de años de estudio por parte de sus autores, los que además tuvieron un papel destacado en el desarrollo histórico de la psiquiatría chilena. Nos interesa analizar particularmente el papel que el desarrollo de la higiene mental comienza a tener en la formulación de nuevas propuestas de cambio de la asistencia asilar. En el segundo apartado se analizan los intentos de institucionalización de la higiene mental en el país, sus principales propuestas e intentos de desplazamiento desde una asistencia psiquiátrica hacia otra de carácter más integral y social.

\section{Propuestas de transformación del modelo asilar}

Para George Rosen, los orígenes del movimiento de higiene mental se encuentran en las propuestas surgidas desde mediados del siglo XIX en torno a la transformación del modelo asilar de asistencia psiquiátrica (Rosen, 1974). En Chile, cuyo primer asilo para orates fue fundado en 1852, las alternativas al manicomio comienzan a proponerse hacia fines de ese siglo. Uno de los primeros en exponer las necesidades de cambio en ese sentido fue el médico residente de la Casa de Orates de Santiago, Manuel Segundo Beca. En 1891, en las Memorias entregadas a la Junta

2 El nombre oficial es Asociación Chilena de Higiene Mental; sin embargo, en algunas publicaciones aparece como Liga Nacional de Higiene Mental. La confusión se produce porque tanto su primer director, Hugo Lea Plaza, como su secretario, Juan Garafulic, usan indistintamente ambos nombres. 
Directiva de la Casa, sostiene la necesidad de "construir un nuevo Manicomio en Santiago, o bien dos en provincias [que] proporcione eficazmente, no solo asilo para estadía y sitio de reclusión o aislamiento de los enajenados, sino residencia hospitalaria y provechosa para su curación" (Medina et al., 2002: 34).

En 1894 la Junta de Beneficencia, el Ministerio del Interior y el Congreso deciden comprar un extenso terreno en Providencia y nombran una comisión compuesta por Germán Greve, Guillermo del Sol, ambos médicos, y por el ingeniero Carlos Barroilhet para que presentara los planos para el Manicomio Nacional que se construiría en ese terreno, creara el Manicomio de Concepción y emprendiera una reforma de la Casa de Orates tendiente a transformarla en hospital psiquiátrico. También se comisionó a Manuel S. Beca para realizar un estudio de los sistemas de asistencia mental en Europa y Estados Unidos. En su informe "Instituciones y servicios de enajenados en Europa y Estados Unidos", entregado al Gobierno y publicado en 1898, defiende detalladamente los beneficios de la transformación del asilo en hospital psiquiátrico, contemplando desde la ubicación y el tipo de construcción hasta la creación de secciones abiertas y salas de observación. Si bien el hospital terminó de construirse cuatro años después de que se adoptara la decisión, inmediatamente fue ocupado por el Ejército chileno y nunca fue devuelto a la Junta de Beneficencia.

El mismo Manuel S. Beca, en el $4^{\circ}$ Congreso Científico de Santiago, realizado entre diciembre de 1908 y enero de 1909, expuso sobre la necesidad de que el personal de servicio de los hospitales psiquiátricos fuera "exclusivamente laico" como ocurre "en todo el mundo civilizado" y sobre la implementación del trabajo al aire libre y de talleres y pequeñas industrias. Respecto a la transformación del asilo propone la instalación de "anexos a los hospitales comunes y destinados exclusivamente al cuidado transitorio de los locos" (Beca, 1910: 173), así como “de locales adecuados para asilar niños y la implementación de talleres para su educación y enseñanza especial" (Beca, 1910: 169).

Germán Greve, psiquiatra chileno, introductor del psicoanálisis en el país, a partir de 1927 comienza una campaña de Higiene Mental a través de las páginas de la Revista de Beneficencia Pública. En los primeros números, posiciona a través de la historia de la psiquiatría la labor del alienista en la dignificación de los locos. En sus primeras editoriales resalta la figura de Pinel y describe con detalle los crueles tratamientos anteriores al surgimiento de la psiquiatría (Greve, 1927b). En los números posteriores desarrolla en extensos artículos los beneficios que reportaría a la higiene mental la construcción de un nuevo Hospital Psiquiátrico; de secciones neuropsiquiátricas en hospitales regionales y la implementación de sistemas de atención de agudos y colonias de puertas abiertas.

En la misma Revista incluye los esquemas que, junto a los otros miembros de la Comisión nombrada por la Junta de Beneficencia, elabora para la construcción de un nuevo Hospital Psiquiátrico. En sendos artículos se refiere a la forma en que la 
arquitectura sanitaria debe ser puesta al servicio de la asistencia de los enajenados. Ya hemos señalado que apenas inaugurado el Hospital fue tomado por el Ejército indefinidamente. También se publican los dibujos de los esquemas de construcción de las futuras secciones neuro-psiquiátricas en los hospitales regionales, las que tampoco se llevaron a cabo.

En 1941, cuando aún no era posible la concreción de un Hospital Psiquiátrico, Greve publica La moderna Asistencia Social Psiquiátrica, donde con un tono marcadamente más pesimista que el que mostraba dos décadas antes, reafirma su compromiso con la "profilaxis mental", reiterando que la asistencia del enajenado continúa siendo una "vergüenza nacional, a pesar de las tantas voces que se han hecho oír para llamar la atención hacia un lamentable descuido de los deberes superiores de Humanidad" (Greve, 1941:6). Sostiene que la implementación de las medidas preventivas debe estar ligada necesariamente "al correcto funcionamiento de las instituciones de Asistencia Social, tanto material como espiritualmente" (Greve, 1941:39), y desplegadas a lo largo “del país entero y no de un conglomerado social humano privilegiado" (Greve, 1941:42).

En 1924, el sub-director y médico jefe de la Casa de Orates Jerónimo Letelier, fue comisionado por la Junta de Beneficencia para estudiar en Europa las colonias agrícolas y los manicomios de puertas abiertas, con la intención de "transformar nuestro antiguo sistema de hospitalización de alienados” (Villaseñor et al., 2011: 163). En su informe, dividido en 5 capítulos (Servicio de Enfermeros; Servicio Médico; Hospitales Urbanos; Hospitales Psiquiátricos y Asilos-Colonias), demandaba la construcción de un nuevo hospital psiquiátrico. En 1929 la Junta de Beneficencia aprobó su construcción, así como la habilitación en locales de emergencia de pabellones psiquiátricos en los hospitales de Iquique, Antofagasta, Viña del Mar y Punta Arenas. Sin embargo, la crisis económica de 1929 paralizó la construcción del Hospital y los pabellones. Hacia fines de la década de 1940, cuando Chile aún no contaba con un Hospital Psiquiátrico nuevo y moderno, Luis C. Muñoz evaluaba la labor de Letelier y de los psiquiatras que le antecedieron en sus propuestas de cambio, como una "vigorosa política médico-psiquiátrica", cuyas propuestas siguen "alumbrando como una norma la solución de nuestros problemas", a pesar de la inactividad del Estado en ese sentido (Muñoz, 1947: 20).

Tanto los testimonios de los actores de los procesos históricos en torno a la salud mental (Marconi, 1998) como la historiografía (Araya, 1998; Araya, Martínez, 2020), han coincidido en la "manicomización" de la asistencia mental en Chile. La transición a un sistema de Hospital General que implicaba la creación de pabellones psiquiátricos para enfermos agudos; la fundación de hospitales psiquiátricos en las principales provincias; la formación de profesionales especializados en psiquiatría como visitadoras sociales o enfermeras psiquiátricas, no se produjo en Chile hasta muy avanzado el siglo veinte y de manera muy parcial. De las propuestas que hemos 
considerado hasta aquí, la única que se concretó en la década de 1920 fue la construcción e inauguración en 1928 de la primera colonia de alienados u opendoor en el fundo El Peral en las afueras de la capital chilena. Sin embargo, rápidamente la Colonia El Peral derivó en un nuevo asilo donde los pacientes eran sometidos a iguales o peores condiciones que los de la Casa de Orates (Gómez y Villanueva, 2010; Araya et al., 2020).

Las propuestas analizadas surgieron exclusivamente desde los psiquiatras. Sus demandas de transformación del asilo en hospital psiquiátrico y la creación de sistemas de puertas abiertas no abogaban por la desinstitucionalización bajo ninguna perspectiva. Como sostiene Rafael Huertas, estamos ante "un intento de modernización que no llegó a cuestionar -a pesar de los servicios de 'puertas abiertas' - ni el hospitalocentrismo del modelo asistencial imperante ni la concepción de tal hospital psiquiátrico como un espacio cerrado de confinamiento". No se trataba, por lo tanto, de echar abajo el asilo sino de potenciar el hospital "medicalizado" (Huertas, 1991: 75). En Chile, si bien las demandas se vinculaban de manera importante a la legitimación de la profesionalización psiquiátrica (Araya, 2018), no es menos cierto que la situación desastrosa de los asilados era una preocupación constante de los alienistas y psiquiatras desde la fundación de la Casa de Orates (Camus, 1993). Aun cuando la fuerza gremial y profesional de los psiquiatras no permitía emplazar al estado, las propuestas analizadas introdujeron en el debate elementos de salud pública como hospital psiquiátrico, hospital de puertas abiertas y pabellones psiquiátricos en hospitales provinciales. La estrategia apuntaba a potenciar a la psiquiatría como parte del incipiente circuito de servicios públicos, extendiendo su acción a la asistencia extrahospitalaria.

\section{Movimiento de Higiene Mental}

En la década de 1930, en sintonía con el movimiento internacional de higiene mental, Chile sumará a las demandas anteriores, frustradas en gran parte, esfuerzos por establecer una Liga de Higiene Mental. Según Gerald Grob (1987), el movimiento de higiene mental surgido en Estados Unidos a fines del siglo XIX obedeció a la vinculación de modos científicos de pensamiento con el poder de organizaciones privadas y el Estado. Las primeras organizaciones higienistas norteamericanas de fines del siglo XIX, como la Asociación Nacional para la Protección de los Insanos y la Prevención de la Insanía (1880), tenían su énfasis en la prevención, pero no lograron vincularse al poder y en poco tiempo desaparecieron. Mientras que el Comité Nacional de Higiene Mental (1909), con financiamiento estatal y sociedades desplegadas en el territorio, tuvo un sostenido crecimiento.

En 1930 se llevó a cabo, en Washington, el Primer Congreso Internacional de Higiene Mental, plataforma para la organización de sociedades y ligas nacionales de 
Higiene Mental en todo el mundo occidental3. El turno de Chile llegó un año después, en 1931, cuando se funda la Asociación Chilena de Higiene Mental, "con un amplio programa que comprendía el estudio y desarrollo de todas las causas sociales susceptibles de producir enfermedades mentales" (Garafulic, 1931: 213). La Liga fue impulsada por médicos, juristas y pedagogos y presidida en su primer directorio por el neurólogo Hugo Lea Plaza, quien se había comprometido un año antes, en la Segunda Conferencia Latinoamericana de Neurología, Psiquiatría y Medicina Legal de Rio de Janeiro a estimular la creación de una institucionalidad en torno a la prevención y el control de las enfermedades mentales en Chile. Hasta Mariano Ruperthuz (2019), la Asociación había sido completamente ignorada por la historiografía. En su investigación, además de proporcionar un análisis detallado de la conformación y de los integrantes de la Asociación, aborda la introducción de los saberes psi en el contexto del desarrollo de la higiene mental.

Luego de la inauguración, su secretario, el médico Juan Garafulic, dicta una Conferencia en la Escuela de Servicio Social donde se refiere en extenso a los objetivos centrales y a los antecedentes que dieron lugar a su creación. Sostiene que la Asociación surge para hacerse cargo, "con formas renovadas de contención", del "aumento extraordinario" de los problemas de "trascendencia social", es decir, aquellos "que por su persistencia e invasión de la colectividad entraba[n] el normal desarrollo físico y espiritual de ella, trayendo como consecuencia una disminución de la capacidad económica de la misma". Garafulic inserta la Asociación en una doctrina política social específica que obedece al contexto de principios de siglo, esto es, "la de que el individuo es un engranaje puesto al servicio de una entidad que puede llamarse patrón o Estado, individuo al que importa conservar, si no se quiere perder la armonía y rendición de la máquina". Esta vez no se trata del "número de locos", sino del aumento de los problemas de "índole social y psicológica", atribuibles al maquinismo, la guerra, la aceleración del ritmo de la vida y la disminución de la seguridad de conservarse orgánicamente (Garafulic, 1931: 212).

Ya no se trata solamente del asilo y del psiquiatra sino del conjunto del tejido social. La desorientación del individuo, provocada por la vida moderna, "da lugar a cavilación de la que se pasa al anulamiento o al trastorno" (Garafulic, 1931: 215). Así, "en su afán de ir a la raíz del mal, la medicina ya no va solamente en pos de los realmente enfermos; ha extendido sus dominios a los simplemente molestos y desorientados, a esa falange de seres que se califican con el nombre de 'nerviosos', 'raros', 'tocados' o con el más genérico de 'histéricos', seres a los que antes se dejaba entregados a sufrirse a sí mismos, o a la rotativa médica irresponsable" (Garafulic, 1931:215). Visto que es toda la sociedad la susceptible de enfermar, la única manera

3 En 1937, en París, se realizan el Segundo Congreso Internacional de Higiene Mental y el Primer Congreso de Psiquiatría Infantil. Se acordó celebrar el tercero en Río de Janeiro, pero el estallido de la guerra lo impidió. 
de limitar los trastornos mentales "es yendo más allá del enfermo, del desequilibrado, o del propenso"; la medicina, "esta vez unida a otras ramas científicas quiere ir lejos, llegar hasta el individuo sano... ¡Qué digo al individuo sano!, más allá aún, al niño, y más aún, al embrión, a evitar que eso que va a 'ser' una persona, llegue ya tarado o predispuesto" (Garafulic, 1931: 215).

La Asociación se asume como un organismo que enfrenta la defensa de la población a través de la "lucha" en diversos frentes: una "lucha psiquiátrica" contra la locura; una "lucha médico-psicológica" contra las alteraciones, desequilibrios y molestias mentales; una "lucha pedagógica" contra "torpes, débiles mentales, tímidos" y, finalmente, una "lucha social-aisladora" contra "locos morales, incorregibles, criminales, etc.” (Garafulic, 1931: 216). Las medidas propuestas abordan la capacidad de adaptación social de los individuos (eugenesia y atención de la infancia); la solución de conflictos psíquicos de acción psicomédica (neurosis, trastornos emotivos, fobias) y de acción pedagógica (retardados y débiles mentales); la prevención de los trastornos psiquiátricos (alcoholismo, enfermedades venéreas); la rehabilitación de los trastornos psiquiátricos a través de la organización eficiente de los hospitales psiquiátricos; y, por último, la instalación de servicios psicológicos en los hospitales y de sanatorios y policlínicos para convalecientes de afecciones mentales ${ }^{4}$.

Para el desarrollo de los dos últimos aspectos, es decir, prevención y rehabilitación de los trastornos psiquiátricos, la Asociación apunta a la construcción de un Hospital Psiquiátrico; la creación de policlínicos; la habilitación de salas para enfermos mentales agudos en Hospitales Generales y la creación de opendoors para los "inaptos para la vida mundana, pero no inservibles del todo para alguna labor vigilada" (Garafulic, 1931: 217). Asimismo, aboga por la multidisciplinariedad, demandando al Estado la introducción de cátedras de psicología o psicopatología. Estas medidas evidencian tanto una continuidad con las propuestas de transformación asilar que los psiquiatras venían articulando desde la última década del siglo XIX, como una propuesta de cambio que busca ampliar el campo de la asistencia meramente psiquiátrica al de la asistencia mental. Esta transición implica además de la integración al proyecto higiénico de educadores, asistentes sociales, pediatras, psicólogos, enfermeras y criminólogos, la participación comprometida del Estado.

Aun cuando la fundación de la Asociación fue un estímulo para la organización de los diferentes actores de la asistencia mental, no logró transformarse en un organismo estatal y tampoco sancionar legislativamente un nuevo modelo de atención. La asistencia a los enajenados se regía por las disposiciones transitorias del Código Sanitario de 1931, que facultaban a la Dirección General de Beneficencia y Asistencia Social para observar, recluir y autorizar la permanencia y salida del Manicomio de los

4 En la última década historiadores como Marcelo Sánchez (2014), Cristián Palacios (2015), César Leyton (2015), Silvana Vetö (2017) y Mariano Ruperthuz (2019), han actualizado las investigaciones en torno a varios de los campos de acción de la higiene mental aquí señalados. 
alienados toxicómanos y presuntos alienados y por el Reglamento de Hospitalización y Reclusión de Insanos de 1927. Se trata de una legislación enfocada en las reclusiones forzosas, bastante lejana aún de los preceptos de defensa de la higiene mental. El médico Elías Malbrán, en 1938, en las Jornadas Neuropsiquiátricas Panamericanas de Lima, demandaba la creación de un Servicio de Higiene Mental en el país, que sancionara la institucionalización de los modelos de asistencia que se venían proponiendo desde los inicios del siglo (Malbrán, 1938: 951).

En 1943, los médicos Isaac Horwitz y Luis Cubillos presentaron en la Primera Jornada Nacional de Neuropsiquiatría celebrada en Viña del Mar, un proyecto de Ley de Alienados que subsanara las deficiencias de la legislación vigente. En 1947, más de 15 años después de fundada la Asociación de Higiene Mental, los mismos médicos presentaron al Congreso Nacional un proyecto de legislación psiquiátrica para la asistencia de los enfermos mentales (Horwitz y Cubillos, 1947). El proyecto, resultado de la discusión de varios anteproyectos elaborados por comisiones de psiquiatras designadas por Salvador Allende en el período en que había sido Ministro de Salubridad, no fue considerado por el Congreso. Para el médico, abogado y diputado Hermes Ahumada Pacheco, los proyectos y propuestas en defensa de la salud mental, "desgraciadamente no han contado ni con la ayuda pecuniaria ni con el entusiasmo y sacrificio de parte de los técnicos" (Ahumada, 1954: 172).

La Asociación Chilena de Higiene Mental dio paso, en 1948, a la Asociación

Chilena Pro-Salud Mental, dirigida por el psiquiatra Arturo Vivado. La nueva organización era filial de la Asociación Latinoamericana de Salud Mental (ALAPROSAM), dependiente de la Federación Mundial de Salud Mental, patrocinada por la OMS, la UNESCO y las Naciones Unidas. Los nueve puntos de su programa muestran una orientación más académica y psiquiátrica que su antecesora. El enfoque está puesto en la educación de la población, en la prevención de los problemas psiquiátricos a través de conferencias de médicos especialistas, en publicaciones en revistas médicas, en intervenciones en radios y en campañas públicas a favor de la construcción de un Hospital Psiquiátrico (Ahumada, 1954: 174-175).

Al menos programáticamente, las ideas de "defensa", "lucha", "vigilancia", así como la incorporación de otras disciplinas a la cruzada por la sanidad mental, aparecen con menor fuerza. De todos modos, ninguna de las dos asociaciones pudo transformarse en organismo estatal. Habrá que esperar hasta 1960 para la creación de la Sección de Salud Mental de la Dirección General del Servicio Nacional de Salud. Esta precaria y tardía institucionalidad es más bien tributaria de la investigación y la epidemiología del alcoholismo desarrollada a partir de la década de 1940 en la Universidad de Chile (Araya y Martínez, 2021). 


\section{Conclusiones}

Desde fines del siglo XIX médicos alienistas viajan a Europa y Estados Unidos a estudiar las transformaciones que están experimentando los clásicos asilos de internación. A su regreso elaboran informes donde proponen para Chile la construcción de un Hospital Psiquiátrico; la habilitación de pabellones psiquiátricos en hospitales generales; la apertura de sistemas de hospitalización del tipo opendoors; la creación de servicios de observación de pacientes; la preparación del personal de servicio en escuelas técnicas y el fomento de la especialización de enfermeras y asistentes sociales en el campo psiquiátrico. Hasta la década de 1940, propuestas similares, pero de nuevos autores, se siguieron sucediendo. El poco éxito de las demandas se verifica en el hecho de que se deberá esperar al menos cinco décadas para la construcción de un nuevo hospital psiquiátrico. Una única colonia opendoor se inaugura a fines de la década de 1920 en la capital chilena, la que se transforma rápidamente en un nuevo asilo tradicional.

Los psiquiatras eran un grupo profesional pequeño. La cátedra se crea en 1927 pero el camino de consolidación fue complejo. Psiquiatras como Arturo Vivado y Luis Custodio Muñoz coinciden en que una de las explicaciones de la débil institucionalización de la asistencia psiquiátrica primero y de la higiene mental posteriormente fue la ausencia de participación de los psiquiatras en la estructura del Estado y en las esferas gubernativas. Evidentemente hay otras razones de importancia, como la falta de tratamientos farmacológicos eficientes que liberaran a los asilados del control y la vigilancia psiquiátrica. Sin embargo, la incorporación al discurso psiquiátrico de la posibilidad de prevención de la locura, de las taras y de los conflictos psíquicos, abrió un derrotero a través del cual los psiquiatras buscaron extender la influencia de la psiquiatría más allá del asilo.

No se trataba de liberar al asilo de la vigilancia médica, pero sí de transformarlo en una institución sanitaria, científica, organizada, eficiente y pública. Para ello incorporaron principios elementales de salud pública como es el desarrollo de distintos niveles de atención de acuerdo a la prevención o la cura. Se propone la instalación de consultas externas, urgencia, dispensarios y pabellones de agudos en hospitales generales. Una organización de esta naturaleza implica tanto la garantía del Estado como el posicionamiento de la psiquiatría al mismo nivel de los otros servicios médicos.

A esta idea de organización psiquiátrica en torno a la prevención de los trastornos mentales se sumará a partir de las primeras décadas del siglo XX el movimiento de higiene mental el que, a través de ligas y asociaciones de médicos, neurólogos, psiquiatras, juristas, pedagogos, voluntarios, grupos filantrópicos, etc., buscará extender la influencia de la medicina mental más allá de los asilos, los hospitales psiquiátricos y los consultorios. A diferencia de las propuestas anteriores, 
que emanaban desde los psiquiatras, el movimiento de higiene mental tenía su base en la comunidad organizada lo que le proporcionaba mejores posibilidades para transformar la asistencia psiquiátrica en un asunto de salud pública.

Es complejo evaluar los resultados del movimiento de higiene mental. En el caso chileno, la constitución de dos asociaciones de ese tipo, más allá de los logros obtenidos, puede considerarse evidencia de la convocatoria y del despliegue público de ciertos grupos académicos. Sin embargo, un movimiento de estas características se despliega en diversos escenarios que exceden lo institucional. Algunos han sido objeto de investigaciones importantes, las que hemos referido en el texto; pero resta aun un amplio campo por explorar. Si bien durante el período estudiado no se logró una sanción legislativa de los cambios que se proponían y la asistencia continuó basada en el viejo asilo decimonónico, hay un desplazamiento del discurso público, favorecido por el desarrollo del movimiento de higiene mental, desde la incurabilidad y el encierro, hacia la prevención y lo comunitario.

\section{Bibliografía}

Ahumada, H. (1954): Plan nacional de defensa de la salud mental. Santiago, Ed. Jurídica de Chile.

Araya, C. (2018): La locura es nuestra: profesionalización de la psiquiatría en Chile. Saberes y prácticas, 1826-1949. Rosario, Prohistoria.

Araya, C.; Morales, N. y C. Leyton (2020): “Archivo del Hospital Psiquiátrico El Peral: una experiencia de investigación desde la historia cultural de la psiquiatría”, Revista de Historia Social y de las Mentalidades, 24(1): pp.147-168.

Araya, C. y C. Martínez (2021): "Epidemiología de los trastornos mentales en Chile y su impacto en las políticas públicas de salud mental, 1950-1973”, Santiago, documento inédito.

Beca, M. (1891): Contribución al estudio de las enfermedades mentales en Chile. Movimiento de la población de la Casa de Orates de Santiago en 1890. Santiago, Imprenta Nacional.

Beca, M. (1898): Instituciones y servicios de enajenados en Europa y Estados Unidos. Santiago, Imprenta Nacional.

Beca, M. (1910): "Estadística de la Locura; Organización de los Asilos de Enajenados. Resultados", en G. Greve, Cuarto Congreso Científico (1 Panamericano) celebrado en Santiago de Chile, del 25 de diciembre de 1908 al 5 de enero de 1909. Trabajos de la 
V Sesión, Ciencias Médicas e Higiene, Tomo II. Santiago, Imprenta Barcelona, pp.164175.

Camus, P. (1993): "Filantropía, medicina y locura: la Casa de Orates de Santiago 18521894", Historia, 27, pp. 89-140.

Garafulic, J. (1931): "Problemas psicológicos actuales", Revista Servicio Social, 5 (3-4), pp. 210-226.

Gómez, M. y M. Villanueva (2010): “Hospital Psiquiátrico El Peral”, en M.A. Armijo, ed., Psiquiatría en Chile. Apuntes para una historia. Santiago, Royal Pharma, pp. 30-47.

Greve, G. (1927): “Felipe Pinel”, Revista de Beneficencia Pública, 11(3): s/p.

Greve, G. (1927b): "Algo sobre los locos y su reclusión y tratamiento con anterioridad a la creación de la Casa de Orates”, Revista de Beneficencia Pública, 11(3): pp.274285.

Greve, G. (1941): La moderna asistencia social psiquiátrica. Santiago, Ediciones de la Universidad de Chile.

Grob, G. (1987): Mental Illnes and American Society, 1875-1940. Princeton, Princeton University Press.

Horwitz, I. y L. Cubillos (1947): "Proyecto de legislación psiquiátrica para la asistencia de los enfermos mentales en Chile", Revista Chilena de Neuropsiquiatría, I, pp.163166.

Huertas, R. (1991): "Salud pública y salud mental: El nacimiento de la política de sector en psiquiatría”, Revista de la Asociación Española de Neuropsiquiatría, 11(37), pp. 73-79.

Huertas, R. (1995): "El papel de la higiene mental en los primeros intentos de transformación de la asistencia psiquiátrica en España”, Dynamis, 15, pp.193-209.

Letelier, J. (1925): Informe sobre la asistencia de los Alienados que presenta a la Honorable Junta de Beneficencia. Santiago, Imprenta Selecta. Reproducido en S. Villaseñor, C. Rojas et al., eds. (2011): Antología de textos clásicos de la psiquiatría latinoamericana. Guadalajara, Grupo Latino Americano de Estudios Transculturales, pp.163-172. 
“Liga Nacional de Higiene Mental” (1931), Revista Servicio Social, 5 (3-4), pp. 262263.

Malbrán, E. (1938): "El Servicio Social en la asistencia de enfermos de la mente en Chile”, II Jornadas Neuropsiquiátricas Panamericanas, Lima.

Marconi, J. (1998): Del asilo a la comunidad: 50 años de psiquiatría chilena, 19481998. Un testimonio personal, Inédito.

Medina, E.; Escobar, E. y M. Quijada, eds., (2002): De Casa de Orates a Instituto Psiquiátrico. Antología de 150 años. Santiago, Sociedad Chilena de Salud Mental.

Molina, C. (2010): Institucionalidad Sanitaria Chilena 1889-1989. Santiago, LOM.

Muñoz, L. (1947): Problemas urgentes de la asistencia psiquiátrica en Chile. Santiago, Imprenta El Imparcial.

Palacios, C.; Leyton, C. y M. Sánchez (2015): Bulevar de los pobres. Racismo, higiene y eugenesia en Chile e Iberoamérica. Siglos XIX y XX. Santiago, Ocho Libros.

Rosen, G. (1974): Locura y sociedad. Sociología histórica de la enfermedad mental. Madrid, Alianza Editorial.

Ruperthuz, M. (2019): “'Saberes psi' y las nuevas psicopatologías a causa de la aceleración del tiempo: las bases para la fundación de la Asociación Chilena de Higiene Mental (1931)", en Hevia, E.; Reiter, F. y G. Salas, eds. Historias de la psicología. Contribuciones y reconstrucciones parciales. Santiago, Ediciones Universidad Alberto Hurtado, pp. 217-253.

Sánchez, M. (2014): “La teoría de la degeneración en Chile”, Historia, 2(47), pp. 375400.

Vetö, S. (2017): "Higiene mental infantil y psicoanálisis en la Clínica de Conducta, Santiago de Chile, 1936-1938”, Asclepio, 69 (2), p. 195.

Fecha de recepción: 13 de octubre de 2020

Fecha de aceptación: 19 de diciembre de 2020 